\title{
Kernos
}

Revue internationale et pluridisciplinaire de religion grecque antique

$17 \mid 2004$

Varia

\section{L'héroïsation du poète Hésiode en Grèce ancienne}

\author{
Marie-Claire Beaulieu
}

URL : http://journals.openedition.org/kernos/1403

DOI : $10.4000 /$ kernos. 1403

ISSN : 2034-7871

Éditeur

Centre international d'étude de la religion grecque antique

Édition imprimée

Date de publication : 1 janvier 2004

ISSN : 0776-3824

\section{Référence électronique}

Marie-Claire Beaulieu, « L'héroïsation du poète Hésiode en Grèce ancienne », Kernos [En ligne],

17 | 2004, mis en ligne le 22 avril 2011, consulté le 02 mai 2019. URL : http://journals.openedition.org/ kernos/1403; DOl : 10.4000/kernos.1403 


\section{L'héroïsation du poète Hésiode en Grèce ancienne'}

Les poètes semblent avoir occupé une place à part dans l'imaginaire grec, car les textes biographiques que nous avons conservés à leur sujet leur attribuent non seulement des innovations artistiques importantes, mais aussi plusieurs aventures et actions d'éclat qui impliquent le contact avec des dieux et des héros. Grâce aux travaux de $M$. Lefkowitz, nous savons maintenant que ces récits ne sont pas authentiques, mais qu'ils constituent seulement le reflet de ce que les ouvres attribuées à ces poètes représentaient pour le peuple grec $^{2}$. Ainsi, il faut voir dans les légendes qui les entourent le statut qu'on leur prêtait : à mi-chemin entre la condition humaine et divine, on croyait qu'ils étaient mêlés de près ou de loin aux affaires des Immortels, tout comme les héros $^{3}$. Ceux-ci recevaient parfois un culte qui se rapprochait peut-être de celui des morts ${ }^{4}$ qu'on aurait également dédié à certains poètes mythiques comme Orphée, Thamyris, Linos, Homère et Hésiode.

Le cas de ce dernier est particulièrement intéressant car les récits qui entourent sa vie et sa mort le relient de nombreuses manières aux sphères héroïque et divine. Quoi d'étonnant si l'on considère l'importance de la poésie traditionnelle transmise sous son nom: bien plus qu'un homme réel possédant une biographie crédible, ce poète est une persona héroïque qui incarne la sagesse presque divine transmise au travers des ouvres qui lui sont attribuées $^{5}$. L'incertitude persiste toutefois en ce qui concerne les termes de

${ }^{1}$ Je tiens à remercier $M$. Pierre Bonnechere du temps et de l'attention qu'il a très généreusement accordés à la préparation de cet article; il m’a aussi fourni plusieurs références bibliographiques et indiqué des pistes de recherche intéressantes. Je remercie également Mme Vinciane Pirenne-Delforge pour ses commentaires et suggestions.

2 M.R. LeFkowitz, The Lives of the Greek Poets, Baltimore, 1981.

3 Au sujet des héros grecs, voir E. KEARNS, «Between God and Man: Status and Functions of Heroes and their Sanctuaries", in Le sanctuaire grec, Entretiens Hardl 37, Genève (1990), p. 6599, ainsi que le volume collectif : V. PIRENnE-Delforge et E. SuÁrez dE LA TORRe (éds), héros et béroünes dans les mytbes et les cultes grecs. Actes du colloque organisé à l'Université de Valladolid du 26 au 29 mai 1999, Liège, 2000 (Kemos supplément 10).

4 C.M. ANTONACCIO, An Archaeology of Ancestors: Tomb Cult and Hero Cult in Early Greece, Lanham, 1995, p. 246-267; C. Sourvinou-INwood, 'Reading' Greek Death. To the End of the Classical Period, Oxford, 1995, p. 87; J.N. Coldstream, "Hero-Cult in the Age of Homer », JHS 96 (1976), p. 8-17.

5 Voir G. NAGY, «Hesiod», in T.J. LuCF (éd.), Ancient Writers, New York, 1982, p. 50 et R. LAMBBerton, Hesiod, New Haven, 1988, p. 28-29. Pour un parallèle avec Homère : J.M. FolEY, "Individual Poet and Epic Tradition: Homer as Legendary Singer», Arethusa 31 (1998), p. 149178. C. BELGER, «Das Grab des Hesiod in Orchomenos ", Berliner Philologische Wochenschrift 12 
son héroïsation, et surtout, du culte qui lui aurait peut-être été rendu. Dans l'espoir de faire la lumière sur cette question, on reprendra ici l'ensemble des sources anciennes pertinentes et on examinera les différentes opinions qui ont été émises à leư sujet par les critiques modernes.

\section{Les récits concernant la mort d'Hésiode}

Les différents récits traditionnels qui entourent la mort d'Hésiode sont de première importance, car ils nous informent sur ce que les Grecs croyaient à propos de sa vie, et pourraient avoir constitué la toile de fond c'un culte hérö̈que". D'abord, le Certamen, qui date du if siècle de notre ère ${ }^{7}$, fait certainement écho à une tradition plus ancienne que l'on retrouvait dans le Mouseion d'Alcidamas du $\mathrm{IV}^{\mathrm{e}}$ siècle av. J.-C $\mathrm{C}^{8}$. De plus, la légende de ce concours poétique semble avoir été connue dès le $\mathrm{v}^{e}$ siècle, selon un passage d'Aristophane et surtout un commentaire de Thucydide?. Tout un corps de contes relié à la vie d'Hésiode avait donc cours au $v^{\mathrm{e}}$ siècle av. J.-C. et sans doute auparavant, ce qui démontre assez l'importance de la persona du poète dans la tradition et dans l'imaginaire collectif grecs ${ }^{10}$.

Le récit de la mort du poète provient fort probablement d'une tradition archaïque $^{11}$ : un fragment d'Héraclite semble montrer que celui-ci connaissait

(1892), p. 98-100, 125 et R.M. CoOK, "Hesiod's Father ", JHS 109 (1989), p. 170-171, considèrent que les détails biographiques que nous possédons au sujet d'Hésiode sont authentiques.

${ }^{6}$ Voir C. CALAME, «Montagne des Muses et Mouseia: la consécration des Travaux et l'héroïsation d'Hésiode », in A. Hurst et A. Schachter (éds), La Montagne des Muses, Genève, 1996, p. 48 : «En effet, comme c'est le cas pour tout poète grec de renom, l'œuvre d'Hésiode a suscité une biographie. Mais cette biographie, avec ses aspects anecdotiques et légendaires relatifs notamment à la mort du poète, n'est que la trace langagière du culte rendu au poète héroïsé. »

${ }^{7}$ La plupart des critiques s'accordent pour dater le Certamen de l'époque de l'empereur Antonin : E. VOGT, "Die Schrift vom Wettkampf Homers und Hesiods », RhM 102 (1959), p. 196; M.L. WEST, «The Contest of Homer and Hesiod», CQ 18 (1967), p. 433; F. Chanoux, «Le poète Hésiode. Esquisse d'une biographie ", in Mélanges offerts en bommage à Étienne Gareau, CEA 14 (1982), p. 14-15.

${ }^{8}$ On considère généralement que l'auteur du Certamen reprenait au moins en partie le Mouseion, car il cite directement Alcidamas à la ligne 240 (Allen).

9 ARISTOPH., La Paix; 1280 et 1286-1287 : ces vers présentent une similitude frappante avec Cert., 205-212 (Allen). Thucrd., III, 96, 1 mentionne la mort d'Hésiode dans le temple de Zeus Néméen pour avoir mal interprété un oracle dont fait mention le Certamen.

10) Cette tradition semble s'être poursuivie durant plusieurs siècles : Eratosthène (c. 275-194 av. J.-C.) aurait écrit une épopée aujourd'hui perdue intitulée Anterinys ou Hésiode à propos de la mort du poète et de la punition infligée à ses meurtiiers. Voir N.j. RICHARDSON, "The Contest of Homer and Hesiod and Alcidamas' Mouseion ", CQ 31 (1981), p. 1.

${ }^{11}$ Nos sources concernant la mort et le premier enterrement d'Hésiode sont : Paus., IX, 38, 34; Cent., 224-236 (Allen); Puut., De l'intelligence des animanx; 969e, 984d; Banquet des sept sages, 162b-e; POLLux, Onomasticon, V 42; Souda, s.v. Hesiodos, 9-11 (Merkelbach-West); THucyd., III, 96, 1; J. TzETż̀s, Vie d'Hésiode, 34-40 (Merkelbach-West). Pour une analyse détaillée de leur interdépendance et de leurs différences, voir O. FrIEDEL, "Die Sage vom Tode Hesiods, nach ihren Quellen Untersucht », Jabrbïcber fïr Classiscbe Pbilologie, Supplement Band 10 (18781879), p. 235-278. 
l'énigme à laquelle Homère ne peut répondre dans le Certamen (323-323 Allen) ${ }^{12}$. On peut donc penser qu'Héraclite connaissait lui aussi le contexte de l'énigme, soit le sujet du Certamen. Et, comme la panhellénisation des épopées homériques et des poèmes hésiodiques date de la période archaïque, de telles élaborations légendaires pourraient remonter à cette époque. Toutes ont le même point de départ, soit le concours poétique tenu à Chalcis entre Homère et Hésiode ${ }^{13}$. Celui-ci, après l'avoir emporté, se serait rendu à Delphes, où la Pythie l'aurait averti d'éviter le sanctuaire de Zeus Néméen, car il y trouverait la mort. Se méprenant sur le sens de l'oracle, un topos en mythologie grecque, Hésiode aurait évité Némée et voyagé plutôt en Locride, ou aux environs de Naupacte ${ }^{14}$, pour mourir assassiné près d'un autre sanctuaire de Zeus Néméen, accusé d'avoir agressé la fille de son hôte ou d'avoir été complice de cette agression ${ }^{15}$. Son cadavre, jeté à la mer, aurait été ramené au rivage par des dauphins lors d'un festival d'Ariane (Certamen) ou de Poséidon (Pausanias). Les gens du pays de Naupacte (Pausanias) ou de Locres (Plutarque, Banquet des sept sages) ou d'Oinoé en Locride (Certamen) ou d'Oineon près de Naupacte (Thucydide) auraient trouvé son cadavre, l'auraient reconnu et enterré avec honneur, soit dans le temple de Zeus Néméen ou dans un endroit secret près du Nemeion (Plutarque, Banquet des sept sages) $^{16}$. Un chien, selon Pollux et Plutarque (De l'intelligence des animaux), aurait désigné les meurtriers par ses aboiements et ceux-ci auraient été punis par le peuple (Plutarque, Banquet des sept sages). Chez Johannes Tzétzès, les meurtriers prennent la fuite par mer et font naufrage dans une tempête. Dans le Certamen, ils prennent la fuite, toujours par mer, et sont foudroyés par Zeus (238-240 Allen).

12 D-K fi: 56 : HipPOL., Refutatio omnium baeresium, 16 (Wendland).

13 Le prix de ce concours aurait été un trépied, consacré par Hésiode aux Muses de l'Hélicon. Plusieurs sources le mentionnent, ainsi que la dédicace (qui varie) gravée sur l'objet: Paus., LX, 31, 3; Ant. Pal. VII, 53; Cert., 213-214 (Allen). Cependant, une recherche récente a montré que Plutarque (Erotikos, $749 \mathrm{~b}$ ), qui rejetait les vers des Travaux et les Jours correspondant à cette joute poétique (646-662), les estimant inatuthentiques, savait que le trépied n'existait que dans la légende. Il contestait donc l'authenticité du trépied que l'on montrait aux visiteurs du Mouseion de l'Hélicon en disant que celui-ci ne servait qu'à vanter l'antiquité du sanctuaire des Muses. Voir R. LAMBfirTON, «Plutarch, Hesiod and the Monseia of Thespiai », ICS (1988), p. 491-504.

14 PAUS., $\mathrm{LX}, 38,3$.

${ }^{15}$ De cette union serait né le poète Stésichore, selon PS.-ARIST., Constitution des Orchoméniens, fr. 565 (Rose) et J. Tzetzìs, Vie d'Hésiode, 37 (Melkerbach-West). Cette association, plus que clouteuse au point de vue chronologique, mais intéressante pour l'étude de la tradition, pourrait s'expliquer par la ressemblance entre les thèmes traités par les deux poètes et la manière assez «épique » qu'a Stésichore d'écrire de la poésie «lyrique » : Frifdel, l.c. (n. 11), p. 266.

16 Plusieurs localités peuvent donc prétendre avoir été le premier lieu d'enterrement d'Hésiode: Oineon, Oinoé, Locres et Naupacte. Des critiques modernes suggèrent également qu'un premier enterrement aurait eu lieu à Ascra, mais aucune source ne permet même de le supposer. Voir R. SCODEL, «Hesiod Redivivus », GRBS 31 (1980), p. 303, n. 7. 
Parmi ces détails ${ }^{17}$, le jet du cadavre à la mer et l'intervention des dauphins revêtent une grande importance, car ils rappellent plusieurs histoires similaires dans lesquelles sont impliqués des héros grecs, comme celle d'Arion le citharède, qui, attaqué sur un bateau, chante le Nome Pytbique puis se jette à la mer pour être sauvé par des dauphins et ramené au Cap Ténare pendant une fête de Poséidon ${ }^{18}$. Plutarque précise qu'à cette occasion, Arion éprouve le désir d'être sauvé, pour acquérir l'apparence d'un favori des dieux et pour qu'ils lui donnent une gloire durable ${ }^{19}$. Le héros lesbien Énalos, quant à lui, se jette à la mer avec une jeune fille sacrifiée à Amphitrite dont il était amoureux, alors qu'un taureau était sacrifié à Poséidon; les amants sont emportés par des dauphins et ainsi préservés de la noyade. De retour à Lesbos, alors qu'une immense vague menace l'île, Énalos est escorté jusqu'au sanctuaire de Poséidon par une troupe de poulpes, ce qui suggère la faveur du dieu ${ }^{20}$. Ino, menacée de mort, saute à la mer avec son jeune fils Mélicerte ${ }^{21}$; elle est par la suite honorée à cet endroit sous le nom de Leukothéa, alors que son fils est transporté par un dauphin à l'Isthme de Corinthe où l'on institue les Jeux Isthmiques en son honneur et où il reçoit un culte héroïque sous le nom de Palaemon ${ }^{22}$. Dans l'Hymne à Dionjysos, les pirates qui avaient tenté de tuer le dieu se précipitent dans les vagues devant sa colère et sont aussitôt transformés en dauphins ${ }^{23}$. Thésée, dans l'Ode XVII de Bacchylide (Snell-Maehler), se jette à la mer pour prouver qu'il est bien le fils du puissant Poséidon, et il est conduit devant Amphitrite et les Néréides par des dauphins, ce qui rehausse son prestige auprès de Minos, qui l'avait mis au défi de prouver ses origines divines.

Ces récits, mis en parallèle avec l'histoire de la mort d'Hésiode, semblent montrer que l'épisode où le corps du poète est jeté à la mer et sauvegardé par des dauphins pourrait signifier qu'il a été hérö̈sé. En effet, tous les personnages mentionnés changent de statut à la faveur du plongeon à la mer, qui les

17 La mort des meurtriers par foudroiement (Certamen) indique l'importance du poète aux yeux de la divinité dans l'imaginaire grec. L'intervention du chien n'est pas non plus dépourvue de signification : comparer plusieurs histoires similaires rapportées par PLuT, De l'intelligence des animaux; 969c-970b.

18 PLuT., Banquet des sept sages, 160e-162b; HÉroD., I, 23-24; ÉLIEN, De la nature des animalux; XII, 45.

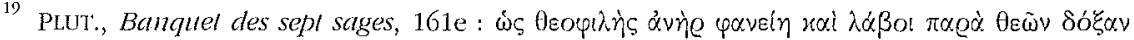
$\beta \varepsilon ́ \beta \alpha t o v$.

20. PLUT., Banquet des sept sages, 163b-d; De l'intelligence des animaux; 984e : Plutarque raconte également l'histoire de l'enfant d'Iasos, qui devint l'ami d'un dauphin et dont le cadavre fut ramené au rivage par celui-ci. Il rapporte aussi en $985 \mathrm{~b}$ que le jeune Télémaque, tombé à la mer, aurait été sauvé par un dauphin; ATHÉN., XI, 466c-d: les dauphins sont absents du récit, mais Énalos nourrit les chevaux de Poséidon.

21 Il n'est pas clair si l'enfant Mélicerte était déjà mort lors du plongeon, car les versions du mythe varient considérablement. Pour un sommaire de la question, voir M. HALM-Tisserant, Cannibalisme et immortalité. L'enfant dans le chaudron en Grèce ancienne, Paris, 1993, p. 178180.

22 Paus., I, 44, 7-9; PluT, Propos de table, 675e-677b.

${ }^{23}$ Hymne à Dionysos VII, 50-53 (Allen). 
met en contact avec les dieux ou avec leurs serviteurs et représentants, les dauphins $^{24}$. Le plongeon constitue donc le moment d'un passage, d'une transformation vers une personnalité plus divine. C'est également le cas pour Hésiode, qui, bien que déjà mort lors de son katapontismos, acquiert tout de même un statut spécial : après que le cadavre du poète eut été enterré avec honneur à l'endroit de sa découverte, l'oracle de Delphes ordonne aux Orchoméniens de le ramener dans leur ville pour enrayer une peste $e^{25}$. Or, nous savons que l'on croyait aux effets bénéfiques des dépouilles de certains héros, comme Oreste et Thésée : on comptait donc Hésiode à leur nombre, à tout le moins dans ce récit ${ }^{26}$. De plus, cette histoire est comparable à celle de Mélicerte-Palaemon, qui, dans la version du mythe que donne Apollodore, est déjà mort lorsque Ino se jette à la mer. Son cadavre, recueilli par des dauphins, est enterré à l'Isthme, où il reçoit un culte héroïque ${ }^{27}$.

Les liens qui unissent plusieurs héros et poètes (surtout Arion et Hésiode) avec les dauphins, sous la gouverne des dieux Poséidon, Dionysos, et parfois Apollon Delphinios apparaissent aussi clairement dans ces légendes ${ }^{28}$. C'est

24 Voir entre autres, C. GaLLINI, “Katapontismós », SWSR 34 (1963), p. 61-90, qui propose de voir dans le plongeon un rite initiatique qui confère son statut d'homme à l'adolescent. Lorsque le mortel est précipité à la mer; comme Mélicerte et Hésiode, selon elle, il renaît en tant que héros ou en tant que myste. Par ailleurs, D. WARLAND, «Que représente la fresque de la paroi Ouest de la tombe «du plongeur " de Poseidonia ?", Kernos 12 (1999), p. 195-206, suggère plutôt que le plongeon représente le passage de la vie à la mort, que transcende la survie de l'âme régénérée par cette calbarsis, parfois en rapport avec le culte d'Apollon, comme à Lencade. Dans cette perspective, l'Hymne à Dionysos pose une difficile énigme que les savants n'ont toujours pas résolue. En effet, la transformation des pirates tyrrhéniens lors du plongeon et leur changement de statut sont évidents, mais le résultat est présenté comme une punition plutôt que comme un acte de reconnaissance divine ou d'initiation.

${ }^{25}$ PS.-ARIST., Constitution des Orchoméniens, fr. 565 (Rose) = J. TzETzÈs, Vie d'Hésiode, 42-44 (Merkelbach-West); Cert., 247-253 (Allen); Ant. Pal. VII, 54.

${ }^{26}$ Voir C. Miralles et J. PÒrtulas, "L'image du poète en Grèce ancienne ", in N. Loraux et C. Miralues (éds), Figures de l'intellectuel en Grèce archailque, Paris, 1998, p. 15-63 (spéc. p. 3637).

27 Selon la version d'Apollodore (III, 4, 3).

28 Dans ce contexte précis, il semble exister un important réseau de liens entre les dieux Poséidon, Apollon et Dionysos, dont les ramifications dépassent de loin les limites du présent travail. En effet, les dauphins sont avant tout les serviteurs de Poséidon et d'Apollon Delphinios. Voir Hymne à Apollon, 400 et 493-496; PuUT., De l'intelligence des animaux; 984c; ÉLEN, De la nature des animaux; XII, 6 et 45. Cependant, ils sont associés à Dionysos cáns l'iconographie: B.S. RidGWAY, «Dolphins and Dolphin-Riders », Arcbaeology 23 (1970), p. 86-95 et S. LATTIMORE, The Marine Thiasos in Greek Sculpture, Los Angeles, 1976. Ce dernier établit des parallèles fort convaincants entre le thiase dionysiaque et le thiase marin, qui forme la suite du dieu Poséidon. De même, Plutarque raconte que le pin, dont on faisait les couronnes des vainqueurs aux Jeux Isthmiques, était à la fois l'arbre de Poséidon et de Dionysos : PLuT., Propos de table, 675e-677b. De plus, ces jeux étaient consacrés à Poséidon et à Palaemon : les deux dieux sont donc étroitement liés aux Jeux Isthmiques ainsi qu'à Palaemon, puisque ce dernier est le frère de lait de Dionysos et que sa mort est liée à Poséidon. L'état actuel de la recherche ne permet pas d'expliquer les détails de cette association entre des divinités fort différentes, mais il semble qu'elle ait été assez importante pour les Grecs, car on la retrouve dans plusieurs sources d'époques différentes. Voir W. BukzerT; Homo Necans (trad. P. Bing), Berkeley, 1983 [1972], p. 203-204 et C.M. BowRA, «Arion and the Dolphin", $M H 20$ (1963), p. 121-134 = On Greek 
que ces animaux possèdent une nature multiple, principalement marine, mais ils appartiennent aussi au monde de la poésie et de la musique, d'où leurs affinités avec les poètes. Comme le précisent Plutarque et Élien, ce sont des serviteurs de Poséidon et d'Apollon Delphinios; ils apprécient l'aulos, les hommes et les dieux, et sont particulièrement chers aux Muses ${ }^{29}$. Athénée affirme qu'ils sont sacrés, sans expliciter davantage leur nature ${ }^{30}$.

\section{Orchomène}

Après sa mort et son premier enterrement, le corps d'Hésiode aurait été transféré à Orchomène, sur ordre de la Pythie ( $x \alpha \tau \dot{\alpha} \chi \varrho \eta \sigma \mu o ́ v)$, parce que la ville était aux prises avec une peste ${ }^{31}$. Plutarque, dans le Banquet des sept sages (162e), rapporte que les Locriens cachèrent les os d'Hésiode dans un endroit secret près du Nemeion pour les soustraire aux prétentions des Orchoméniens; cependant, le texte n'indique pas si cette tentative de dissimulation fut un succès. Si l'on en croit Pausanias (IX, 38, 2-4), les Orchoméniens trouvèrent tout de même l'endroit en question, grâce à la Pythie et à l'aide d'une corneille, et ensevelirent les restes du poète chez eux ${ }^{32}$. Ils déposèrent la dépouille dans la tholos d'Orchomène, tombe du héros fondateur Minyas ${ }^{33}$.

On peut admettre deux interprétations, dont la première paraît être la plus plausible. En effet, les faits rapportés par les sources, particulièrement Pausanias qui est bien connu pour ce genre de récit, font songer à une légende étiologique : celle-ci devait expliquer l'existence de deux tombeaux d'Hésiode, ce que la translation des reliques justifiait aisément. Les Orchoméniens auraient senti le besoin, et ceci à une époque qui peut être aussi bien haute que tardive, d'expliquer la présence de la grande tholos dans leur ville et de lui associer des événements merveilleux (cf. le récit prodigieux de la construction de la tholos, Pausanias, IX, 38, 2-3) ${ }^{34}$. D'autres ont préféré conclure que les Orchoméniens avaient réellement voulu posséder ces précieuses reliques, associées à celles de leur héros fondateur Minyas, dans le but de rehausser leur prestige civique à une époque où ils voulaient dominer les

\footnotetext{
Margins, 1970, p. 164-181. Celui-ci est d'avis que l'homme sauvé par les dauphins est toujours, sauf dans le cas de Télémaque, un substitut de Poséidon, car il lui est toujours relié. Cependant, Hésiode et Arion font songer à Dionysos et Apollon (Arion chante le Nome Pythique avant de plonger à la mer), alors qu'Énalos rappelle plutôt Poséidon et que Palaemon est lié autant à ce dernier qu'à Dionysos.

29 PLUT., De l'intelligence des animaux, 984 C; ÉLIF, De la nature des animaux; XII 6 et 45. Voir aussi PIND., fr: $140 \mathrm{~B}$ Snell-Maehler, analysé par W.J. HENDERSON, "Pindar fr. 140B SnellMaehler: the Chariot and the Dolphin », Hermes 120 (1992), p. 148-158.

3i) ATHÉN., Les Deipnosophistes, 282e-f.

31 Cf. supra, n. 25.

32 À propos de la comeille, Voir W. Schiller, "Die Tiere beim Tode Hesiods », Anthropos (1934), p. 812-814.

33 Johannes Tzetzès se borne à indiquer "le milieu de l'agora ».

${ }^{34}$ Voir A. SCHAChter, Cults of Boiotia, 2. Herakles to Poseidon, Londres, 1986 (BICS, Suppl. 38.2), p. 143-144. Le chercheur croit qu'il est possible que la «tombe » ne se soit trouvée à cet endroit que pour le bénéfice des visiteurs.
} 
villes béotiennes environnantes. Selon R.J. Buck, ce transfert aurait eu lieu avant la fin clu vII" siècle av. J.-C., quand les gens d'Ascra se seraient enfuis à Orchomène en emportant les os d'Hésiode ${ }^{35}$ en raison de la prise de contrôle de leur village par les Thespiens ${ }^{36}$. P. Wallace croit plutôt que le transfert daterait du milieu du $v^{e}$ siècle av. J.-C., parce que les Orchoméniens occupaient à cette époque une place prépondérante au sein de la confédération béotienne, et la possession des os du poète les aurait aidés à se maintenir dans cette position ${ }^{37}$. Cependant Pausanias, qui fait le récit le plus détaillé de ce transfert émaillé de faits merveilleux, ne donne aucune information quant au temps écoulé entre le premier et le second enterrement, et aucune autre source ne contient d'informations à ce propos. Donc, dans le cas d'une éventuelle utilisation historique de ces documents, sans pouvoir nier la possibilité d'un transfert réel des reliques, la première interprétation des faits semble de loin préférable, surtout si l'on considère que nous n'en possédons aucune preuve matérielle ou épigraphique.

En effet, nous n'avons à ce jour pas de preuves concrètes de l'héroïsation d'Hésiode: les sources archéologiques font défaut, les fouilles de la tholos d'Orchomène n'ayant pratiquement rien livrée ${ }^{38}$. Les objets trouvés, épars et mal conservés, ne permettent pas d'affirmer qu'un culte aurait été célébré dans la tholos, sauf aux périodes hellénistique et romaine, et rien ne permet de les associer à Hésiode ${ }^{39}$. Quoi qu'il en soit, par son caractère grandiose, ce monument est un endroit susceptible de donner lieu à un culte qui pourrait, entre autres possibilités, relever de légendes étiologiques. Il faut également garder en mémoire le fait que toutes les communautés humaines ont besoin d'un passé dont se réclamer: c'est ainsi que l'on a réutilisé des tombes mycéniennes à des époques ultérieures comme lieux de culte dédiés à des

35 Aucune source ne mentionne l'existence d'un culte d'Hésiode à Ascra bien que plusieurs critiques l'aient pris pour acquis sur la base du témoignage du Ps.-ARIST., Constitulion des Orcboméniens, fr. 565 (Rose), comme Friedel, l.c. (n. 11), p. 235-278, et plus récemment, NAGr, l.c. (n. 5), p. 50-51.

36 R.J. BuCK, A History of Boeotia, Edmonton, 1979, p. 98.

37 P. WALlACE, "The Tomb of Hesiod and the Treasury of Minyas at Orchomenos", in J.M. Fossey et H. Grroux (éds), Actes du troisième congrès intennational sur la Béotie antique/ Proceedings of the Third Intemational Conference on Boiotian Antiquities, Amsterdam, 1985 , p. 167.

${ }^{38}$ Malgré le peu de crédit généralement accordé à $H$. Schliemann, il faut s'en remettre à son article «Exploration of the Boiotian Orchomenus» IHS 2 (1881), p. 122-163 pour plusieurs informations concernant la tholos. Cependant, il est indispensable de consulter les précisions apportées par $O$. PELON, Tholol, tumuli et cercles funéraires. Recherches sur les monuments funèbres de plan circulaire dans L'Égée de lâge du Bronze, Paris, 1976, p. 237. Pour un sommaire récent des connaissances et une liste des objets trouvés, voir ANTONACCIO, o.c. (n. 4), p. 127-130.

39) Voir Coldstream, l.c. (n. 4), p. 11. Ce chercheur voit dans le matériel trouvé par Schliemann "a massive evidence for cult", parce qu'on y trouve des tessons d'époque mycénienne, hellénistique et romaine. Cependant, peut-on vraiment dire que l'évidence est "massive »? La rareté du matériel trouvé et son hétérogénéité ne permettent pas une telle conclusion, bien qu'il paraisse évident qu'il y a eu un culte tardif d'un dieu ou d'un héros, qui nous reste inconnu, dans la tholos. 
héros ou aux ancêtres ${ }^{40}$. Le fait que l'on ait cru qu'il s'agissait de la tombe de Minyas est aussi très significatif : la tholos constitue un monument honorifique voué non seulement à ce héros, mais à toute la cité, et est donc doublement sacrée $^{4 t}$. De plus, il est certain qu'un culte dédié au héros en question s'y tenait, parce que des jeux célébrés à cet endroit, les Minyeia, sont attestés; il est cependant impossible de savoir si ce culte comportait d'autres aspects ${ }^{42}$.

Par ailleurs, plusieurs auteurs ont conservé une épigramme élégiaque qui aurait figuré sur le tombeau d'Hésiode à Orchomène ${ }^{43}$. Les différentes sources présentent quelques variantes mineures, mais le contenu en est toujours le même. Voici la version que transmet Pausanias, qui précise que l'inscription se trouvait sur la tholos (IX, 38, 4):

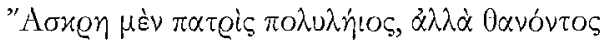

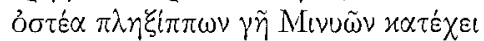

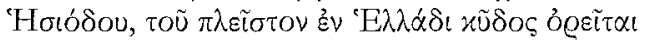

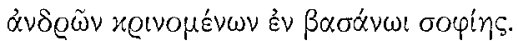

Askra est sa patrie aux nombreux champs de blé, mais mort, la terre des Minyens dompteurs de chevaux recouvre les os d'Hésiode, à qui appartient une très grande gloire en Grèce, parce que les hommes le jugent au critère de la sagesse ${ }^{4 i}$.

Bien entendu, on pourrait y voir une preuve du transfert de la dépouille d'Hésiode à Orchomène, et peut-être même de son ensevelissement dans la tholos. Mais il faut se garder d'une interprétation si catégorique : l'inscription pourrait très bien avoir été gravée, en toute bonne foi, dans la foulée de la croyance aux légendes étiologiques qui entouraient la tholos, et/ou dans le désir des Orchoméniens de prétendre posséder les os d'Hésiode de manière à augmenter leur prestige politique et culturel auprès des autres cités béotiennes. En somme, la seule conclusion définitive que l'on peut en retirer est que la ville d'Orchomène était certainement l'un des foyers de la popularité d'Hésiode à l'époque de Pausanias, et que cela remontait au moins à la période

40 J. WHITLEY, "Tomb Cult and Hero Cult: the Uses of the Past in Archaic Greece», in N. SPENCer (éd.), Time, Tradition and Society' in Greek Archaeology, Londres / New York, 1995, p. 49.

Voir Sourvinou-Inwood, o.c. (n. 4), p. 87. À ce sujet, C. Picard a fait l'hypothèse que l'ensevelissement d'Hésiode en terre sacrée aux côtés de Minyas avait servi à accorder au poète les honneurs dus aux fondateurs de cités ("L'inhumation ad sanctos dans l'antiquité \#, RA 27 [1947], p. 83). Toutefois, il est nécessaire de se demander si, par cette réelle ou légendaire inhumation, on a voulu honorer Hésiode ou la cité qui a accueilli son corps.

${ }^{42}$ Paus., LX, 38, 3; POxy' XXVI, 2451A fr. 1 col. II, 27 et suiv. : ce fragment, daté du I ${ }^{\mathrm{cr}}$ siècle de notre ère, ou du début du II $^{\complement}$, préserve des bribes d'un commentaire sur les Istbmiques de Pindare, dans lequel on précise que les Minyeia avaient lieu à Orchomène. Voir SCHACHTER, o.c. (n. 34), p. 143.

43 Paus., IX, 38, 4; J. TzETzÈs, Vie d'Hésiode, $45-48$ (Merkelbach-West) = Ps.-ARIST., Constitution des Orcboméniens, fr. 565 (Rose); Cert., 250-253 (Allen); Ant. Pal. VI, 54 (attribue l'épigramme à Mnasalcée, un poète élégiaque du II" siècle av. J.-C).

Les traductions sont miennes. 
hellénistique. Tant l'héroïsation du poète que l'existence d'un culte y sont, pour le moment, invérifiables.

\section{Le mont Hélicon}

Outre la ville d'Orchomène, le mont Hélicon a également retenu l'attention des chercheurs ${ }^{45}$. Sur les pentes de cette montagne se trouvait un sanctuaire important consacré aux Muses où l'on célébrait les Mouseia ${ }^{46}$. Selon Pausanias, le sanctuaire comprenait un bois sacré ( $\alpha \dot{\lambda} \lambda \sigma o \varsigma)$ où l'on pouvait admirer plusieurs statues aujourd'hui perdues qui représentaient les Muses, leur nourrice Euphémé (sur un bas-relief), les poètes Thamyris et Linos, Apollon et Hermès, Dionysos (qui avait là deux statues), l'aulète Sacadas d'Argos, Orphée de Thrace avec Télété, Arion de Méthymne chevauchant un dauphin et Hésiode tenant une cithare sur ses genoux ${ }^{47}$. Il semble significatif de retrouver Arion et son clauphin parmi les représentations de tant d'autres poètes, car on connaît l'importance des dauphins dans les légendes concernant Hésiode. De plus, Sacadas est un aulète, et l'on sait que les Grecs croyaient ces animaux sensibles à l'instrument. Les dieux Dionysos et Apollon, patrons de la poésie et de la musique, sont aussi représentés dans la grotte : le dauphin est donc entouré de personnages avec lesquels il est fréquemment associé. 'Toutefois, il est important de conserver' en mémoire qu'il ne s'agissait pas de la statue d'un dauphin seul, mais bien de celle d'Arion chevauchant l'animal : la présence du dauphin peut donc simplement renvoyer à la légende qui entoure ce poète, ou refléter tous les liens énumérés précédemment. La présence des autres personnages est d'importance capitale, car il s'agit pour la plupart de héros poètes qui recevaient un culte, ou qui à tout le moins étaient liés à un culte, comme Linos ${ }^{48}$, Thamyris ${ }^{19}$ et Orphée : ceci donne encore plus de crédit à l'hypothèse d'une héroïsation d'Hésiode ${ }^{5 i !}$.

${ }^{45}$ L’Hélicon a été exploré par des philologues classiques désireux d'identifier les lieux décrits par Hésiode et de vérifier son témoignage au sujet du climat d'Ascra. Voir P. WALLACE, "Hesiod and the Valley of the Muses ", GRBS 15 (1974), p. 5-24. Cette recherche n'a que peu d'interetêt scientifique si l'on considère que les commentaires d'Hésiode à ce sujet et les détails «biographiques » qu'il foumit se trouvent dans un contexte poétique: il ne s'agit pas de décrire des lieux réels mais bien de produire un effer poétique. Voir M. GRIFFITH, "Personality in Hesiod", Classical Antiquity 2 (1983), p. 37-65.

46 Nos sources littéraires concernant les Mouseia sont : Paus., LX, 31, 3; Pollux, Onomasticon, I 37; pour les inscriptions, Voir SchachTER, o.c. (n. 34), p. 163-179.

47 PaUs., IX, 29, 5.

18 PAUS., IX, 29, 6.

49) On retrouve en effet un groupe de symthytai Thamymridontes à Thespies dans la première moitié du IV" siècle av. J.-C. Voir P. RoEsch, Etudes béotiennes, Paris, 1982, p. 162.

${ }^{50}$ Cependant, certains donnent trop d'importance à ces associations, allant jusqu'à dire que parce qu'Hésiode est associé à Linos, qui recevait des sacrifices au Mouselon, il y aurait aussi eu un sacrifice à Hésiode près de la source Hippociène, qui n'est aucunement mentionnée par les témoignages anciens comme lieu possible de sacrifices dédiés au poète. Voir CALAME, l.c. (n. 6), p. 54. 
Venons-en à une táblette de plomb vue par Pausanias près de la source Hippocrène sur laquelle on avait gravé Les Travaux et les Jours en amputant l'œuvre de son prologue ${ }^{51}$. C. Calame et G. Nagy ont soutenu que la mention de cette tablette prouve l'existence d'un culte d'Hésiode étant donné que l'on a gravé de telles tablettes pour consacrer les ouvres d'Orphée, Sophocle, Archiloque et l'Hymme bomérique à Apollon dans des temples et que ces poètes recevaient un culte héroïque au moins à l'époque hellénistique ${ }^{52}$. G. Nagy ajoute que la nature de ces cultes est profondément archaïque, sans préciser en quoi cela consiste ${ }^{53}$ : bien qu'il soit très probable que certains de ces cultes remontent à la période archaïque, toutes les attestations que nous en possédons remontent au plus à la période hellénistique. L'inscription de Mnésiepes, dont Nagy se sert pour prouver l'existence d'un culte d'Archiloque à Paros, date du III $^{e}$ siècle av. J.-C. Bien que le parallèle soit intéressant et offre sans conteste une explication plausible au témoignage de Pausanias, il est nécessaire de garder en mémoire que, si l'on a gravé la tablette de l'Hélicon dans le but de la consacrer aux Muses ou à toute autre divinité, le texte de Pausanias n'y fát aucunement allusion et nous n'en avons aucune autre trace; or, Pausanias signale souvent le culte des héros. De plus, même si la tablette a bel et bien été consacrée aux: Muses, peut-on vraiment dire qu'elle constitue une preuve suffisante pour en conclure qu'il y avait un culte d'Hésiode à cet endroit?

Il ne serait franchement pas impossible qu'Hésiode ait été associé à des manifestations religieuses sur l'Hélicon, soit au Mouseion ou près de la source Hippocrène. Une borne trouvée à Thespies et datée de 230 av. J.-C. en constitue un indice, mais tellement énigmatique qu'il est encore une fois difficile d'en tirer plus que des suppositions. Voici l'inscription telle que la restitue W. Dittenberger (IG VII, 1785):

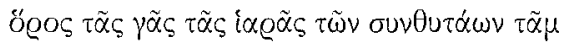

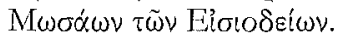

Ceci est la borne du terrain consacré des Hésiodiens qui sacrifient ensemble (synthytai) aux Muses ${ }^{51}$.

L'interprétation de cette inscription est extrêmement délicate : en effet, elle montre clairement que, dans cette région et à l'époque hellénistique, un groupe de personnes pratiquant un certain culte qui impliquait des sacrifices était réuni autour des Muses et se réclamait d'Hésiode. Mais, la borne étant l'unique attestation connue de l'existence de cette association religieuse, on ne peut en dire beaucoup plus. T.W. Allen a cru qu'ils formaient une école de

51 PAUS., LX, 31, 4.

52 Calane, l.c. (n. 6), p. 55-56; NAGY, l.c. (n. 5), p. 51. Pour une analyse du culte dédié à Sophocle et des remarques concernant celui voué à Archiloque, voir A. CONNOLr, "Was Sophocles Heroised as Deixion? ", JHS 98 (1998), p. 1-21.

53 NAGY, l.c. (n. 5), p. 51 .

5 La traduction de l'inscription est controversée, mais il semble sûr que le génitif masculin

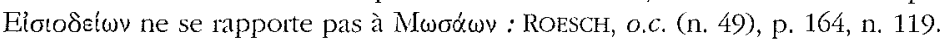


rhapsodes similaire à celle des Homeridai mais la pierre elle-même n'en fournit aucune preuve et nous manquons d'autres sources ${ }^{55}$. De plus, il n'est question que de sacrifices aux Muses, et non à Hésiode : le nom des Hésiodiens est le seul indice que foumit la borne à propos d'un culte du poète. En conséquence, le prudent commentaire de P. Roesch paraît de loin supérieur : les synthytai, dont il existe plusieurs autres exemples contemporains en Béotie, sactifiaient ensemble à une divinité comme l'indique leur nom, et, dans le cas présent, il s'agit des Muses. Quant au rôle joué par Hésiode dans les activités de la confrétie, l'inscription n'en donne aucun détail ${ }^{56}$. En somme, il n'y a donc pas ici matière à affirmer qu'il s'agit d'une preuve tangible d'un culte d'Hésiode. Les parallèles fournis par P. Roesch permettent toutefois de remettre cette association religieuse en contexte, c'est-à-dire à une époque et dans une région où les groupes de synthyytai étaient assez nombreux et où ils semblent avoir eu assez d'importance pour posséder un terrain consacré.

Une stèle de marbre ornée d'une figure d'homme barbu a aussi été trouvée sur l'emplacement d'Haghia Trias, sur les pentes de l'Hélicon ${ }^{57}$. On a cru qu'il s'agissait d'Hésiode, ou d'un Cyclope, ou d'un Bergdämon, ou encore d'une personnification de l'Hélicon ${ }^{58}$. Il est toutefois difficile d'y voir plus qu'une dédicace d'un certain Euthyclès aux Muses, qui fait référence à Hésiode comme étant un maître de sagesse et de paix. Les inscriptions qu'elle porte ne semblent en rien liées à un culte d'Hésiode, mais bien plutôt à celui des Muses à travers le respect dû à Hésiode qui les avait honorées. La mention de la grande sagesse du poète montre encore une fois son importance dans la littérature et l'imaginaire des Grecs, particulièrement à l'époque hellénistique ${ }^{59}$.

55 Selon ce chercheur, l'un de leurs enseignements consistait à dire que de tout le corpus hésiodique, seulement Les Iravaux et les jours était authentique, et encore, amputé de ses dix premiers vers. Il tente d'expliquer de cette façon ce que rapporte Pausanias en $\Gamma X, 31$, 4, soit qu'on avait gravé Les Travaux el les Jours en omettant le prologue sur une tablette de plomb dont il a déjà été question: T.W. Allen, Homer: the Origins and the Transmission, Oxford, 1924, p. 48.

56 RoEsch, o.c. (n. 49), p. 162-165. Les autres associations de symbylai attestées dans la région, dont aucune n'est plus ancienne que le $\mathrm{VV}^{\mathrm{e}}$ siècle av. J.-C, sont répertoriées par Roesch à la page 162. Entre autres, on retrouve des synthytai qui ont dédicacé une inscription à Athéna à Thèbes et Thespies au $\mathrm{V}^{\prime \prime}$ siècle av. J.-C., d'autres à Tanagra qui ont enterré l'un des leurs sous le titre de syntbytai d'Aphrodite et d'Aristée au début du II $^{4}$ siècle av. J.-C. et, ce qui est plus important pour notre propos, des synthytai ont dédicacé au poète Thamyris à Thespies au début du $\mathrm{V}^{*}$ siècle av. J.-C. Ce type d'association est donc connu pour au moins un autre poète associé à Hésiode.

57 IG VII, 4240, $\mathrm{HI}^{\circ}$ siècle av. J. $\cdots$ C.

58 Voir P. JAMOT, "Stèle votive trouvée dans l'bieron des Muses », BCH 14 (1890), p. 546-551; W. PEEK., "Hesiod und der Helikon ", Philologus 121 (1977), p. 173-175; A. Hurst, « La stèle de l'Hélicon ", in HuRST - SCHACHTER, o.c. (n. 6), p. 57-72.

59) Voir les parallèles fournis par G. ARGOUD, "L'Hélicon et la littérature grecque », in HurST SCHACHTER, o.c. (n. 34), p. 57-72 et M.L. WEST, "A New Poem about Hesiod», ZPE 62 (1984), p. 33-36; voir aussi Ant. Pal. II, 12; VII, 52; IX, 24; IX, 64. 


\section{Épigiaammes}

Enfin, Johannes Tzetzès a conservé une inscription qui aurait figuré sur le tombeau d'Hésiode à Orchomène, en précisant que Pindare en aurait été l'auteur ${ }^{611}$. Elle est difficile à dater, mais il n'est pas impossible qu'elle remonte à la période archaïque selon ce que l'on peut tirer de sa grammaire, de sa syntaxe et de son vocabulaire ${ }^{61}$.

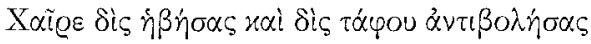

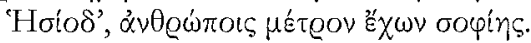

Salut à toi Hésiode, qui as été jeune deux fois et qui as été deux fois au tombeau

Possédant pour les hommes la mesure de la sagesse.

L'interprétation de l'épigramme est très difficile, particulièrement parce qu'il est malaisé de savoir à quoi elle se réfère lorsqu'il est question de deux jeunesses et de deux mises au tombeau. K.J. McKay est d'avis qu'il s'agit d'une vie et d'une vieillesse extraordinaires exprimées dans les termes fabuleux d'Hésiode. En effet, celui-ci avait la réputation d'avoir vécu jusqu'à un âge très avancé et d'avoir été actif dans les deux phases de sa vie : dans sa première jeunesse, il avait été paysan et père d'un fils, et dans la deuxième, après sa rencontre avec les Muses, il avait été poète et père de Stésichore ${ }^{62}$. Cette interprétation est tout à fait plausible, car elle tient compte des légendes qui circulaient à propos de la vie d'Hésiode : l'épigramme pourrait facilement s'inscrire dans cette tradition.

R. Scodel, de son côté, si elle croit que les deux mises au tombeau se réfèrent aux deux enterrements rapportés par la tradition, suggère que les deux jeunesses constituent une allusion à une seconde vie. ${ }^{6.3}$. Elle met donc l'épigramme en parallèle avec plusieurs traditions concernant le retour à la vie ou le rajeunissement de dieux et de héros dont le corps avait été démembré, comme Dionysos-Zagreus chez les Orphiques et Pélops. La formulation d'un passage de Lycophron à propos de celui-ci (Alexandria, 157), rappelle même l'épigramme attribuée à Pindare: on y dit que Pélops est sic $\hat{\eta} \beta \hat{\eta} \sigma \alpha \nu \tau \alpha$ « qui a eu deux jeunesses »; plusieurs autres des exemples cités comportent aussi des termes similaires. Toutefois, peut-on vraiment comparer la tradition concernant la mort d'Hésiode avec celles concernant la résurrection ou le rajeunissement de héros et de dieux démembrés, même s'il existe un certain parallélisme au niveau des mots utilisés pour décrire le phénomène en question?

(11) J. TzErzżs, Vie d'Hésiode, 49-51 (Merkelbach-West); PS.-Aust., Constitution des Orcboméniens, fr. 565 (Rose). Le fragment d'Aristote ne mentionne toutefois pas l'auteur de l'épigramme.

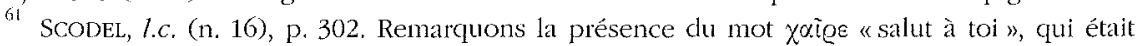
utilisé à la période archaique pour s'adresser aux défunts héroïsés. Voir Sourvinou-INwood, o.c. (n. 4), p. 187-195.

62 K.J. MCKAY, "Hesiod's Rejuvenation », CQ9 (1959), p. 1-5. Cette interprétation expliquerait

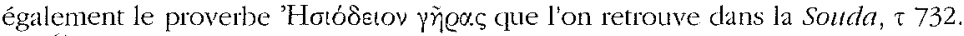

${ }^{63}$ SCODEL, l.c. (n. 16), p. 307-308. 
Les sources ne mentionnent rien de tel, et ne permettent pas non plus de supposer qu'il existait une tradition selon laquelle Hésiode serait revenu à la vie après sa mort ou qu'il aurait eu une seconde vie. Cette épigramme attribuée à Pindare en serait le seul témoignage, si vraiment on peut voir une allusion certaine à une seconde vie ou à une résurrection dans les mots $\delta$ ic $\hat{\eta} \beta \dot{\eta} \sigma \alpha \nu \tau \alpha$.

R. Scodel compare aussi l'épigramme qui nous occupe avec certains passages de l'Héraclès d'Euripide qui concernent la katabasis du héros et son retour au monde des vivants : selon elle, ces passages se réfèrent clairement à la métempsycose, par comparaison avec la katabasis d'Orphée, parce qu'ils contiennent des allusions à une seconde jeunesse ${ }^{\text {gí }}$. La seconde jeunesse que souhaite obtenir le chœur (656-666), dans le contexte de la descente d'Héraclès aux Enfers, serait identique à une seconde vie obtenue par le retour du royaume d'Hadès. L'allusion de l'épigramme à la seconde jeunesse d'Hésiode signifierait donc que le poète serait revenu d'entre les morts, ce qui le lierait au mouvement orphico-pythagoricien et le placerait au nombre des « chamans grecs » bien connus comme Pythagore, Épiménide et plusieurs autres, qui étaient aussi revenus à la vie après leur mort réelle ou supposée. L'attribution des vers à Pindare, bien connu pour ses liens avec le mouvement orphico-pythagoricien, remonterait peut-être à une époque où cette allusion à la métempsycose était encore comprise ${ }^{65}$. Cependant, il est difficile de relier Hésiode, Héraclès, Orphée et Pythagore, puisque rien dans les nombreux textes qui nous sont parvenus à propos du poète d'Ascra, incluant l'épigramme attribuée à Pindare, ne laisse croire qu'il existait une tradition concernant une quelconque katabasis. Remarquons aussi que la métempsycose ne constituait pas un concept unifié dans l'Antiquité : les croyances à ce sujet différaient selon les mouvements religieux et se sont aussi transformées avec le temps ${ }^{\left(\sigma_{6}\right.}$. Il est donc difficile d'associer automatiquement toute allusion à une seconde vie, s'il est bel et bien question de cela, à la métempsycose ou à un quelconque mouvement qui aurait enseigné cette doctrine. En somme, il est donc beaucoup plus probable que l'épigramme attribuée à Pindare par

6 SCODEL, l.c. (n. 16), p. 308-314.

65 Sconel, l.c. (n. 16), p. 320. Toutefois, on voit mal pourquoi il faudrait que l'épigramme soit très ancienne pour remonter à une époque où l'allusion à la métempsycose était encore comprise: l'orphisme, un des mouvements qui enseignaient cette doctrine, est attesté dès la période archaïque jusqu'à l'époque romaine.

б5 voir L. ZHMUD, Wissenscbaf, Pbilosopbie und Religion im fiäben Pybagoreismus, Berlin, 1997; C. CALANE, "Orphismus ", ONP, 2000; H. LLOYD-JONES, "Pindar and the After-Life ", in Pindare, Genève, 1985 (Entretiens sul'Antiquité classique, 31), p. 245-279; W. BuRKRT, Lore and Science in Ancient Pythagormism (trad. E.L. Jr. Minar), Cambridge, 1972, P. 120-165; W.K.C GuTHRIE, Orphée et la religion grecque. Etude sur la pensée orpbique (trad. S.M. Guillemin), Paris, 1956; H.S. LONG, A Study of the Doctrine of Metempsychosis in Greece fiom Pylhagoras to Plato, Phd. Diss., Princeton, 1948; M.P. NILSSON, "Eally Orphism and Kindred Religious Movements ", HThR 28 (1935), p. 181-230. Pour la différence entre les doctrines, comparer, par exemple, PLATON, Ménon, 81b-c, PIND., O/. II, 57-67 et les graffiti orphiques analysés par L. ZHimu, "Orphism and Graffiti from Otbia », Hemes 120 (1992), p. 159-168. 
Johannes Tzetzès se réfère aux deux enterrements d'Hésiocle et/ou aux deux phases bien distinctes de sa vie, ou encore à une subtilité qui nous échappe.

Enfin, soulignons l'importance d'une épigramme élégiaque attribuée à Alcée de Mytilène (c. 620 av. J.-C.) ou à Alcée de Messène (fl. 200 av. J.-C.) par l'Antbologie Palatine VII, 55 :

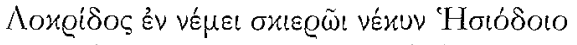

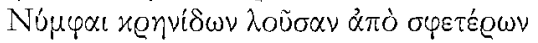

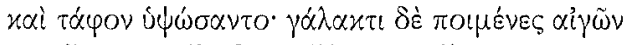

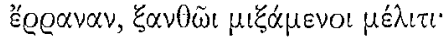

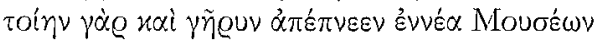

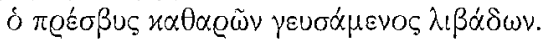

Dans un pré ombragé de Locride les Nymphes lavèrent le cadavre d'Hésiode avec de l'eau de leurs sources et lui élevèrent un tombeau. Sur celui-ci les chevriers versèrent des libations de lait mélangé à du miel doré. Car telle était la voix que le vieillard avait exhalée, lui qui avait goûté aux pures fontaines des neuf Muses.

On peut l'interpréter, en toute prudence, de deux façons: d'abord, la Locride comme lieu de sépulture s'inscrit très bien dans la tradition, qu'on fasse référence au premier enterrement d'Hésiode ou encore à la seconde inhumation à Orchomène ${ }^{67}$. Ensuite, on précise bien dans l'épigramme que les chevriers font des libations de miel et de lait. Or, le miel est connu pour ses propriétés divinatoires et ses liens avec la poésie ${ }^{68}$, et il pouvait être mélangé

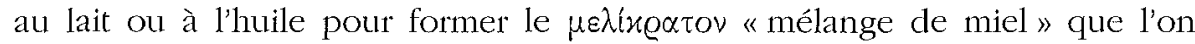
répandait lorsqu'on offrait des libations sans vin appelées nephalia. Les circonstances de cette pratique, qui s'est perpétuée de l'époque archaïque jusqu'à la période romaine, ne sont pas très claires. Il semble que l'on dédiait ces libations surtout aux morts et aux dieux chtoniens ainsi qu'à certains héros ${ }^{(19}$. On peut donc dire qu'il n'est pas impossible que l'on fasse ici allusion à un culte dédié à Hésiode même si l'évidence est des plus minces. Il est en outre malaisé de savoir si Hésiode aurait reçu des sacrifices de ce type en sa qualité de défunt ou à titre de héros ou si l'épigramme illustre seulement une possibilité cultuelle que la fonction poétique (et donc divine) d'Hésiode

${ }^{67}$ Dans ce cas, la légende étiologique concernant l'enterrement d'Hésiode dans la grande tholos d'Orchomène constituerait une tradition limitée à cette localité, comme cela était fréquent dans l'Antiquité.

68 Voir, entre autres, Hymne bomérique à Hermès, 550-563 (Allen); la poésie est comparée au miel chez Hés., Tbéog., 84, Pind., Pyth. X, 53, Swon., fr. 88 (Page) et Platon, Ion, 534a-b.

6) Par exemple: SopH., Ét., 894 (libations de lait sur la tombe d'Agamemnon); PLuT, Le démon de Socrate, 578e (libations de lait sur un tombeau); Esch., Les Perses, 613 (libations de miel et de

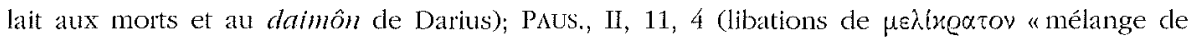
miel » aux Érinyes); HOM, 11 . XXIII, 170-221 (Achille dépose des jarres de miel et d'huile sur le tombeau de Patrocle et ensuite fait des libations de vin en invoquant son âme). Voir F. GraF, "Milch, Honig und Wein. Zum Vertändnis der Libation im griechischen Ritual", in Perennitas. Studi in onore di Angelo Brelich, Rome, 1980, p. 209-221 et A. VERBANCK-PIÉRARD, "Héros attiques au jour le jour : les calendriers des dèmes ", in V. PIrENNE-DELForge (éd.), Les panthéons des cités des origines à la Périégèse de Pausanias, Liège, 1998 (Kennos suppl. 8), p. 119-120. 
semblait commander d'elle-même $e^{70}$. De plus, la nature de ce culte resterait très imprécise, puisque, s'il semble avoir été assez répandu dès l'époque archaïque, le culte des morts et ses rituels particuliers nous sont en grande partie inconnus ${ }^{71}$.

\section{Conclusion}

Le réseau d'informations formé par les diverses sources semble au total suffisamment dense pour justifier une affirmation voulant qu'Hésiode ait été héroïsé. Il occupait une place intermédiaire entre les dieux et les hommes dans l'imaginaire grec, ce qui est démontré, entre autres, par la légende de l'intervention des dauphins lorsque son cadavre a été jeté à la mer et le parallèle avec plusieurs récits similaires concernant les héros Arion, Énalos, Palaemon et Thésée. Il n'est pas impossible qu'un culte héroïque lui ait été rendu, sous forme de sacrifices ou de nephalia, mais les sources ne nous en fournissent aucune preuve définitive. Au mieux, on peut en conclure qu'il est vraisemblable qu'Hésiode a pu être associé à une certaine forme de culte dans certaines régions, en accord avec un arrière-fond de croyances qui présenterait le poète comme divinement inspiré. Dans cette perspective, nous pouvons dire que le personnage d'Hésiode héroïsé a eu, comme de nombreux autres héros et dieux, des attributions diverses à différents endroits et à différentes époques, et ce n'est nullement étonnant : la persona du poète, en tant qu'elle est un produit de la tradition, est sujette à des variations au sein de la mouvance de cette même tradition.

Marie-Claire BEAULIEU

University of Texas at Austin

Department of Classics

1 University Station \#C3400

AUSTIN, Texas 78712

77) A. Henrichs précise en effet qu'il n'y a généralement aucun moyen de savoir avec certitude pourquoi certains dieux et certains héros recevaient des nephalia plutôt que des libations de vin. Selon lui, le culte des héros, en tant qu'extension du culte des morts, ne requérait pas absolument l'exclusion du vin, car on offrait du vin pur à certains motts et à certains dieux chtoniens. Voir "The 'Sobriety' of Oedipus. Sophocles OC 100 misunderstood », ISPP 87 (1983), p. 87-100.

71 Pour un sommaire récent des connaissances dans ce domaine, voir ANTONACCIO, o.c. (n. 4); SOURVINOU-INWOOD, o.c. (n. 4); Whitley, l.c. (n. 40). 